\title{
Coulomb-nuclear interference effects in proton-proton scattering: A simple new eikonal approach
}

\author{
Loyal Durand $\odot^{*}$ \\ Department of Physics, University of Wisconsin, Madison, Wisconsin 53706, USA \\ Phuoc Ha® ${ }^{\dagger}$ \\ Department of Physics, Astronomy and Geosciences, Towson University, Towson, Maryland 21252, USA
}

(Received 15 July 2020; accepted 13 August 2020; published 31 August 2020)

\begin{abstract}
We present a simple new approach to the treatment of Coulomb-nuclear interference and form-factor effects in high-energy proton-proton scattering in the context of an eikonal model for the spin-averaged scattering amplitude. We show that the corrections to the nuclear and Coulomb amplitudes do not depend sensitively on the details of the eikonal amplitude and can be taken as universal, and present parametrizations for the necessary corrections. We also present a simple model for the nuclear scattering amplitude useful for data analysis at small momentum transfer which builds in the proper nuclear phase and the diffraction zeros in the real and imaginary parts of the amplitude.
\end{abstract}

DOI: 10.1103/PhysRevD.102.036025

\section{INTRODUCTION}

The interplay of Coulomb and nuclear interactions in the scattering of charged particles has been studied by many authors ([1-9] and further references therein) with particular emphasis on the use of Coulomb-nuclear interference effects to determine the real part of the nuclear scattering amplitude in proton-proton and proton-antiproton scattering at high center-of-mass energies $W=\sqrt{s}$ and small squares of the covariant momentum transfer $q^{2}=|t|$. The results most used in recent analyses of spin-averaged high-energy data appear to be those of Cahn [3] as modified by Kundrát and Lokajiček [5]. In their approach, the Coulomb and purely nuclear effects are separated out in a spin-independent scattering amplitude, with its components expressed in terms of convolutions involving the nuclear and Coulomb amplitudes with the effects of the proton electromagnetic form factors included. The result is rather cumbersome to use, especially because the way in which the amplitudes are separated leaves long-range effects associated with the Coulomb amplitude in some terms. Questions have also

\footnotetext{
*ldurandiii@comcast.net; Mailing address: 415 Pearl Ct., Aspen, CO 81611.

pdha@towson.edu

Published by the American Physical Society under the terms of the Creative Commons Attribution 4.0 International license. Further distribution of this work must maintain attribution to the author(s) and the published article's title, journal citation, and DOI. Funded by SCOAP.
}

been raised about the treatment of the form factors $[10,11]$; see also $[12,13]$ for further references.

In the present paper, we present an analysis of the Coulomb and form-factor effects in $p p$ scattering based on an eikonal model for the spin-averaged $p p$ scattering amplitude in the limit in which spin effects can be ignored, as discussed in the Appendix. Possible spin effects were considered in detail by Buttimore, Gotsman, and Leader in [6] using a small- $|t|$ or small- $q^{2}$ expansion. We show in the Appendix that these effects are likely to be negligible at the energies of current interest in $p p$ scattering, $W \gtrsim 100 \mathrm{GeV}$.

Our eikonal approach is based on a realistic model which fits the $p p$ and $\bar{p} p$ data from $4.5 \mathrm{GeV}$ to cosmic ray energies, and is consistent with the phase constraints imposed by analyticity [14]. The model allows us to calculate the Coulomb and form-factor effects in the scattering without significant approximation at any value of $q^{2}$ for which it holds, extending beyond the first diffraction minimum in the differential cross section. No small- $q^{2}$ expansion such as those used in [6] and, over an extended range, in [7], is necessary.

Our separation of the various effects in the scattering is different from that of Cahn [3], with long-range effects appearing only in a pure Coulomb scattering term with unmodified form factors, and - to high accuracy - with the remaining effects isolated in a nearly model-independent phase factor that modifies the purely nuclear term,

$$
f\left(s, q^{2}\right) \approx-\frac{2 \eta}{q^{2}} F^{2}\left(q^{2}\right)+e^{i \Phi_{\mathrm{tot}}\left(s, q^{2}\right)} f_{N}\left(s, q^{2}\right) .
$$


As is usual in the treatment of infinite-range Coulomb scattering, an irrelevant overall phase has been absorbed. In contrast to [6], we can treat the various corrections to the spin-independent terms completely, without their expansion in $t$ and $\alpha \ln |t|$.

The advantage of this form of the amplitude is that the Coulomb term is real, making it clear that the Coulombnuclear interference depends only on the real part of the second term, that is, on the real part of $f_{N}\left(s, q^{2}\right)$ with a (small) admixture of the imaginary part dependent on the phase $\Phi_{\text {tot }}\left(s, q^{2}\right)$. The latter is essentially model independent for any eikonal model consistent with the measured $p p$ total cross sections, the forward slope parameters $B=-d \log \left(d \sigma / d q^{2}\right) / d q^{2}$, and the diffractive structure at larger $q^{2}$. We find that $\Phi_{\text {tot }}\left(s, q^{2}\right)$ is small and easily parametrized in the $q^{2}$ region inside the first diffraction zero.

In the following sections, we first discuss the separation of the various effects in the scattering in Sec. II A, and then evaluate the Coulomb scattering term in eikonal form in Sec. II B, and the form-factor effects in Sec. II C. We show, in particular, that the Coulomb and form-factor effects combine to high accuracy to give a combined amplitude of the form $-\left(2 \eta / q^{2}\right) F_{Q}^{2}\left(q^{2}\right) e^{i \Phi_{c, F F}}$, where $\eta=\alpha / v \approx \alpha$, $F_{Q}\left(q^{2}\right)$ is the charge form factor of the proton, and $\Phi_{c, F F}$ is a known phase.

We evaluate the remaining nuclear-dependent term in Sec. II D, where we show that the effects of the Coulomb interaction and the form factors combine at small $q^{2}$ to simply multiply the nuclear amplitude by a phase factor $e^{-\Delta \Phi\left(s, q^{2}\right)}$. The Coulomb and modified nuclear amplitudes can then be combined in the form in Eq. (1), with an overall phase absorbed. We also present an accurate-and essentially model-independent-parametrization of the phase $\Phi_{\text {tot }}$ in Eq. (1) valid from energies below $100 \mathrm{GeV}$ to $20 \mathrm{TeV}$ or above. The result is a very simple implementation of the Coulomb and form-factor corrections in the complete scattering amplitude.

In Sec. III, we apply these results to analyze a model used in recent fits to Coulomb-nuclear interference at very high energies $[13,15]$ in which the phase of the nuclear amplitude is taken as constant. We show that this leads to effective real parts of the scattering amplitude significantly larger than the actual real parts. It is very simple to improve the model at high energies by using an approximate nuclear phase that takes into account the zeros in the real and imaginary parts of $f_{N}\left(s, q^{2}\right)$ which lie within or close to the region in $q$ used in the fits, and give parametrizations of the locations of those zeros in our eikonal model valid from $\sim 500 \mathrm{GeV}$ to above $20 \mathrm{TeV}$.

Finally, for completeness, we discuss the connection of the spin-averaged scattering amplitude to the complete spin-dependent elastic scattering matrix in the Appendix.

\section{ANALYSIS}

\section{A. Background}

In the absence of significant spin effects-thought to be very small at high energies - the spin-averaged differential cross section for proton-proton scattering can be written in terms of a single spin-independent amplitude,

$f\left(s, q^{2}\right)=i \int_{0}^{\infty} d b b\left(1-e^{2 i\left(\delta_{c}^{\mathrm{tot}}(b, s)+\delta_{N}(b, s)\right)}\right) J_{0}(q b)$,

as sketched in the Appendix. Here $q^{2}=-t$ is the square of the invariant momentum transfer, $b$ is the impact parameter, $\delta_{c}^{\text {tot }}(b, s)$ is the full Coulomb phase shift including the effects of the finite charge structure of the proton, $\delta_{N}(b, s)$ is the nuclear phase shift, and

$$
\delta_{c}^{\mathrm{tot}}(b, s)=\delta_{c}(b, s)+\delta_{c}^{F F}(b, s),
$$

where $\delta_{c}$ gives the phase shift for a pure Coulomb interaction, and $\delta_{c}^{F F}$ accounts for the effects of the form factors at large momentum transfers or short distances. This form, with (approximately) additive phase shifts, and that in Eq. (2), can be derived in the context of potential scattering through a Glauber-type treatment [16] of the eikonal function.

With our normalization, the differential elastic scattering amplitude is

$$
\frac{d \sigma}{d q^{2}}\left(s, q^{2}\right)=\pi\left|f\left(s, q^{2}\right)\right|^{2} .
$$

The finite proton charge structure appears through the charge form factors $F_{Q}\left(q^{2}\right)$ measured in electron-proton scattering [17]. Only the charge form factor appears. The magnetic moment scattering with form factor $F_{M}\left(q^{2}\right)$ appears only in the spin-dependent part of the scattering amplitude and does not contribute to Coulomb-nuclear interference in the spin-averaged cross section except through interference effects in the average of the spindependent terms, thought to be very small and neglected here; see, e.g., [6,18]. This is discussed further in the Appendix. The exact spin-averaged Coulomb amplitude for proton-proton scattering should therefore reduce to

$$
f_{c}^{B}\left(s, q^{2}\right)=-\frac{2 \eta}{q^{2}} F_{Q}^{2}\left(q^{2}\right)
$$

in Born approximation.

Equation (2) can be rearranged in the form

$$
\begin{aligned}
& f\left(s, q^{2}\right) \\
& =f_{c}\left(s, q^{2}\right)+i \int_{0}^{\infty} d b b e^{2 i \delta_{c}(b, s)}\left(1-e^{2 i \delta_{c}^{F F}(b, s)}\right) J_{0}(q b) \\
& +i \int_{0}^{\infty} d b b e^{2 i \delta_{c}(b, s)+2 i \delta_{c}^{F F}(b, s)}\left(1-e^{2 i \delta_{N}(b, s)}\right) J_{0}(q b) .
\end{aligned}
$$


Here $f_{c}\left(s, q^{2}\right)$ is the Coulomb amplitude without form factors. The second term in Eq. (6), which we will label $f_{c}^{F F}$, accounts for the effects of the form factors on the Coulomb scattering, strongly reducing the $1 / q^{2}$ falloff of the pure Coulomb term at large $q^{2}=|t|$. The final term $f_{N, c}$ includes the effects of the nuclear scattering as modified by the Coulomb and form factor effects. We will consider these individually in the following subsections. The pure nuclear amplitude $f_{N}\left(s, q^{2}\right)$ is just

$$
f_{N}\left(s, q^{2}\right)=i \int_{0}^{\infty} d b b\left(1-e^{2 i \delta_{N}(b, s)}\right) J_{o}(q b) .
$$

\section{B. Coulomb scattering}

The Coulomb phase shift depends on the parameter $\eta=z_{1} z_{2} \alpha / v, v=2 p W /\left(W^{2}-2 m^{2}\right)$ the velocity of either particle in the rest frame of the other, here expressed in terms of the total center-of-mass energy $W=\sqrt{s}$ and the corresponding proton momentum $p=\sqrt{W^{2} / 4-m^{2}}$; clearly $\eta \approx \alpha \ll 1$ for protons at high energies. The phase shift for the orbital angular momentum $L$ is given to leading order in $\alpha$ by ([19], Sec. 14.6)

$$
\begin{aligned}
e^{2 i \delta_{c}} & =\frac{\Gamma(L+1+i \eta)}{\Gamma(L+1-i \eta)}=\exp \left(2 i \sum_{k=1}^{L} \arctan \frac{\eta}{k}\right) \\
& =\exp \left(2 i \sum_{k=1}^{L}\left(\frac{\eta}{k}-\frac{1}{3} \frac{\eta^{3}}{k^{3}}+\cdots\right)\right) \\
& \approx \exp 2 i\left(\eta \ln L+\eta \gamma+O\left(\frac{\eta}{L}, \eta^{3}\right)\right)
\end{aligned}
$$

Thus, for $L=p b$ large, $b$ the impact parameter in the scattering, the Coulomb phase factor to first order in $\eta$ is

$$
e^{2 i \delta_{c}(b, s)}=e^{2 i \eta(\ln p b+\gamma)}=(p b)^{2 i \eta} e^{2 i \eta \gamma},
$$

where $\gamma=0.5772 \ldots$ is Euler's constant.

The result for $f_{c}(s, t)$ follows from Eq. (2) for pure Coulomb scattering, $\delta_{c}^{F F}=\delta_{N}=0$. This gives

$$
\begin{aligned}
f_{c}(s, t) & =i \int_{0}^{\infty} d b b\left(1-e^{2 i \delta_{c}(s, b)}\right) J_{0}(q b) \\
& \longrightarrow-i e^{2 i \eta \gamma} \int_{0}^{\infty} d b b(p b)^{2 i \eta} J_{0}(q b),
\end{aligned}
$$

where we have dropped the delta function in $q$ associated with the 1 in the first term in the integrand and restricted our attention to angles away from the extreme forward direction, $q>0$. This is the usual restriction for Coulomb scattering, necessitated by the infinite range of the interaction and the associated failure of the term $e^{2 i \delta_{c}}$ to vanish for $b \rightarrow \infty$ ([19], Sec. 14.6).
To evaluate the apparently divergent integral in Eq. (11), we note that the phase factor in Eq. (8) and the scattering amplitude in Eq. (11) are analytic in $\eta$ and the phase is nonsingular and nonzero for $|\Im \eta|<L+1$ or $|\Im \eta|<p b+1$. We can therefore take $\Im \eta \gtrsim \frac{1}{2}$ initially for $p p$ scattering $(\eta=+\alpha / v)$. The integral then converges and gives [Eq. (10.22.43) of [20]]

$$
\begin{aligned}
f_{c}(s, t) & =-\frac{2 \eta}{q^{2}}\left(\frac{4 p^{2}}{q^{2}}\right)^{i \eta} e^{2 i \eta \gamma} \frac{\Gamma(1+i \eta)}{\Gamma(1-i \eta)} \\
& =-\frac{2 \eta}{q^{2}}\left(\frac{4 p^{2}}{q^{2}}\right)^{i \eta}=-\frac{2 \eta}{q^{2}}\left(\frac{1-\cos \theta}{2}\right)^{-i \eta},
\end{aligned}
$$

where we have expanded the ratio of gamma functions $\Gamma(1 \pm i \eta)$ to first order in $\eta$. The result is analytic in $\eta$, and can be continued back to $\Im \eta=0$, giving the usual Coulomb amplitude, but with the relativistic rather than nonrelativistic value of $\eta$.

We can obtain the same result by introducing a convergence factor $e^{-a b}$ in the integrand for $\eta$ real and then using the second form of the result in Eq. 13.2(3) of [21]. This gives

$$
\begin{aligned}
\int_{0}^{\infty} & d b b^{1+2 i \eta} e^{-a b} J_{0}(q b) \\
= & \frac{1}{\left(a^{2}+q^{2}\right)^{1+i \eta}} \Gamma(2+2 i \eta) \\
& \times{ }_{2} F_{1}\left(1+i \eta,-\frac{1}{2}-i \eta ; 1 ; \frac{q^{2}}{q^{2}+a^{2}}\right) .
\end{aligned}
$$

Taking the limit $a \rightarrow 0$ using the limiting form of the hypergeometric function for unit argument and the duplication formula for gamma functions reproduces the result in Eq. (12).

Cahn [3] obtained a similar result with the factor $(2 p / q)^{2 i \eta}$ replaced by $(\lambda / q)^{2 i \eta}$ through somewhat loose arguments by starting with a screened Coulomb interaction with $1 / q^{2} \rightarrow 1 /\left(q^{2}+\lambda^{2}\right)$ in Born approximation. The difference is a phase factor $e^{2 i \eta} \ln 2 p / \lambda$ with a phase which diverges for $\lambda \rightarrow 0$. This is a standard problem. The same phase appears in all terms in Eq. (6), so the phase does not affect the cross section and can be absorbed if it is done consistently in Eq. (6). This phase is model dependent in general. Bethe, for example, uses a Gaussian cutoff in Eq. (4.28) of [1]. Islam [4] used a similar form to evaluate the Coulomb amplitude. The results in both cases differ from that in Eq. (12) only by (different) infinite phases in the Coulomb limit.

As noted above, magnetic-moment scattering does not contribute to the spin-independent part of the scattering amplitude. Its contribution to the spin-dependent 
amplitude is also suppressed by an angular factor proportional to $\sqrt{q^{2}}$ at small $q^{2}$. The factor $1 / q^{2}$ from the photon propagator therefore partially cancels and the amplitude is suppressed relative to the charge-scattering term for $q^{2} \rightarrow 0$. The magnetic form factors $F_{M}^{2}\left(q^{2}\right)$ further suppress the scattering at large $q$. As a result, the magnetic terms do not contribute significantly to the scattering in the region of interest here. This result is general, and holds also for scattering through higher multipoles in the case of particles of higher spin (Secs. 4b and 4c of [18]). The potential interference effects of the magnetic terms with spin-dependent terms in the nuclear scattering studied in [6] are also expected to be negligibly small at high energies as discussed in the Appendix.

\section{Form-factor corrections to Coulomb scattering}

We turn now to the evaluation of the second integrals in Eq. (6) which contains the effects of the form factors on the Coulomb scattering. We will use the standard form

$$
F_{Q}\left(q^{2}\right)=\frac{\mu^{4}}{\left(q^{2}+\mu^{2}\right)^{2}}
$$

for the proton charge form factor with $\mu^{2}=0.71 \mathrm{GeV}^{2}$. We will write the $q^{2}$-dependent factors in the initial Born amplitude as

$$
\begin{aligned}
\frac{\alpha}{q^{2}} F^{2}\left(q^{2}\right) & =\frac{\alpha}{q^{2}}-\frac{\alpha}{q^{2}}\left(1-\frac{\mu^{8}}{\left(q^{2}+\mu^{2}\right)^{4}}\right) \\
& =\frac{\alpha}{q^{2}}-\sum_{m=0}^{3} \alpha \frac{\mu^{2 m}}{\left(q^{2}+\mu^{2}\right)^{m+1}} .
\end{aligned}
$$

Applying an inverse Bessel transform to the second term, which we identify as the leading term in an expansion of the eikonal in terms of $2 \delta_{c}^{F F}$, gives

$$
2 \delta_{c}^{F F}=-\sum_{m=1}^{4} \frac{\alpha}{2^{m} \Gamma(m+1)}(\mu b)^{m} K_{m}(\mu b)
$$

as the eikonal function for the form-factor corrections, where we have used the result in Eq. (10.22.46) of [20] and the symmetry $K_{-\nu}(z)=K_{\nu}(z)$ in evaluating the integrals.

Substituting the form-factor term in Eq. (6) and expanding to leading order in $\delta_{c}^{F F}$, allowed because the form-factor terms are small and compact in impact-parameter space, we obtain a sum of integrals of the form

$$
\begin{aligned}
& \frac{1}{2^{m} \Gamma(m+1)} e^{2 i \eta \gamma} \int_{0}^{\infty} d b b(p b)^{2 i \eta}(\mu b)^{m} K_{m}(\mu b) J_{0}(q b) \\
& \quad=\frac{1}{\mu^{2}}\left(\frac{2 p}{\mu}\right)^{2 i \eta} e^{2 i \eta \gamma} \frac{\Gamma(m+1+i \eta) \Gamma(1+i \eta)}{\Gamma(m+1)}{ }_{2} F_{1}\left(m+1+i \eta, 1+i \eta ; 1 ;-\frac{q^{2}}{\mu^{2}}\right) \\
& \quad=\left(\frac{4 p^{2}}{q^{2}+\mu^{2}}\right)^{i \eta} e^{2 i \eta \gamma} \frac{\mu^{2 m}}{\left(q^{2}+\mu^{2}\right)^{m+1}} \frac{\Gamma(m+1+i \eta) \Gamma(1+i \eta)}{\Gamma(m+1)}{ }_{2} F_{1}\left(m+1+i \eta,-i \eta ; 1 ; \frac{q^{2}}{q^{2}+\mu^{2}}\right),
\end{aligned}
$$

where the result follows from Eq. 13.45(1) of [21] and a standard linear transformation on the resulting hypergeometric function.

The parameter $\eta$ is very small. Setting it equal to zero in the remaining hypergeometric function, expanding appropriately elsewhere, and summing over $m$, we find that the form-factor correction to the leading Coulomb amplitude is

$$
\begin{aligned}
& -\sum_{m=0}^{3} \alpha\left(\frac{4 p^{2}}{q^{2}+\mu^{2}}\right)^{i \eta} \frac{\mu^{2 m}}{\left(q^{2}+\mu^{2}\right)^{m+1}} \\
& =-\frac{\alpha}{q^{2}}\left(\frac{4 p^{2}}{q^{2}+\mu^{2}}\right)^{i \eta}\left(1-\frac{\mu^{8}}{\left(q^{2}+\mu^{2}\right)^{4}}\right)[1+O(\eta)] .
\end{aligned}
$$

Supplying the necessary overall factors and adding the unmodified Coulomb term, the full Coulomb amplitude with the form-factor corrections becomes

$$
\begin{aligned}
f_{c}\left(s, q^{2}\right)+f_{c}^{F F}\left(s, q^{2}\right) \\
=-\frac{2 \eta}{q^{2}}\left(\frac{4 p^{2}}{q^{2}}\right)^{i \eta}\left[1-\left(\frac{q^{2}}{q^{2}+\mu^{2}}\right)^{i \eta}\right. \\
\left.\quad+\left(\frac{q^{2}}{q^{2}+\mu^{2}}\right)^{i \eta} \frac{\mu^{8}}{\left(q^{2}+\mu^{2}\right)^{4}}\right] .
\end{aligned}
$$

The form-factor corrections do not have the simple form of the pure Coulomb amplitude multiplied by $F^{2}\left(q^{2}\right)$ assumed, for example, by Cahn [3] when the phase factors are included. However, the limiting behaviors of the full amplitude are evident from Eq. (21). For $q^{2} \ll \mu^{2}$, the last two terms in this expression cancel, and the full result approaches the pure Coulomb amplitude as expected from the infinite range of that interaction. In the opposite limit, $q^{2} \gg \mu^{2}$, the first two terms eventually cancel to an extra $O(\eta)$, while the last term approaches the pure Coulomb 
result multiplied by $F^{2}\left(q^{2}\right)$. All terms are important for intermediate $q^{2}$.

We can see the structure of Eq. (21) more clearly by looking separately at the amplitude and phase of the factor which multiplies the Coulomb amplitude $-\frac{2 \eta}{q^{2}} e^{i \eta \ln 4 p^{2} / q^{2}}$ :

$$
\begin{gathered}
\operatorname{mag}[\cdot]=\frac{\mu^{8}}{\left(q^{2}+\mu^{2}\right)^{4}}\left[1+O\left(\eta^{2}\right)\right] \\
\arg [\cdot]=-\arctan \left[\eta\left(\frac{\left(q^{2}+\mu^{2}\right)^{4}}{\mu^{8}}-1\right) \ln \frac{q^{2}}{q^{2}+\mu^{2}}\right] \\
=-\eta\left(\frac{\left(q^{2}+\mu^{2}\right)^{4}}{\mu^{8}}-1\right) \ln \frac{q^{2}}{q^{2}+\mu^{2}} \\
\times\left[1+O\left(\eta^{2} \frac{\left.\left(q^{2}+\mu^{2}\right)^{4}\right)}{\mu^{8}}\right)\right] .
\end{gathered}
$$

The approximation in the last line is valid in the region of small $q^{2}$ where Coulomb-nuclear interference is significant, with $q^{2}<\mu^{2}$.

We remark that other parametrizations of the proton charge form factor consistent with the dispersion relations for that quantity-expressions involving sums or integrals of inverse powers of quantities $\left(q^{2}+\lambda^{2}\right)$-lead to results of the same general form, but involving further sums or integrals. The differences among the common parametrizations are unimportant.

Using the results in Eqs. (21)-(23), we obtain finally for the form-factor corrections to the Coulomb amplitude for $\eta / F_{Q}^{2}\left(q^{2}\right) \ll 1$,

$$
\begin{gathered}
f_{c}\left(s, Q^{2}\right)+f_{c}^{F F}\left(s, q^{2}\right)=-\frac{2 \eta}{q^{2}} F_{Q}^{2}\left(q^{2}\right) e^{i \Phi_{c, F F},} \\
\Phi_{c, F F}\left(s, q^{2}\right) \approx \eta \ln \frac{4 p^{2}}{q^{2}}-\eta\left(\frac{\left(q^{2}+\mu^{2}\right)^{4}}{\mu^{8}}-1\right) \ln \frac{q^{2}}{q^{2}+\mu^{2}},
\end{gathered}
$$

where we have used the (standard) parametrization for charge form factor in Eq. (15).

\section{Coulomb and form-factor corrections to the nuclear amplitude}

We turn next to the final term in Eq. (6), the amplitude for the nuclear scattering including the effects of the Coulomb phase shifts and the form-factor corrections,

$$
\begin{aligned}
f_{N, c}\left(s, q^{2}\right)= & +i \int_{0}^{\infty} d b b e^{2 i \delta_{c}(b, s)+2 i \delta_{c}^{F F}(b, s)} \\
& \times\left(1-e^{2 i \delta_{N}(b, s)}\right) J_{0}(q b),
\end{aligned}
$$

where $\delta_{c}(b, s)=\eta \ln p b+\eta \gamma$ and $\delta_{c}^{F F}(b, s)$ is given in Eq. (17). The form-factor effects can be isolated subject to Coulomb and nuclear corrections by expanding to first order in $\delta_{c}^{F F}$, but we have not found this to be especially useful. The Coulomb effects are of course long range, and a similar expansion of those terms is not useful.

A simple approximation to the integral in Eq. (27) is to replace the Coulomb and form-factor phases in that expression by their values at the peak of the uncorrected eikonal distribution, and factor the resulting constant phase out of the integral. This approximation, originally suggested by Bethe [1], works well for the slowly varying Coulomb factor. It is less accurate when the form-factor term is included as that expression varies significantly over the same range in impact parameter as the eikonal term itself, and affects the value of the integral. The dependence of the Bessel function $J_{0}(q b)$ in Eq. (27) also introduces strong dependence on $q^{2}$ as that quantity increases, leading to diffraction zeros in the real and then the imaginary parts of the amplitude, so the Bethe approximation is only valid at small $q^{2}$.

We note for completeness that the location of the peaks in both the imaginary and real parts of the nuclear distribution is at $b_{\text {peak }} \approx \sqrt{\sigma_{\text {tot }} / 4 \pi}$ at $q^{2}=0$ [14]. The $q^{2}$ dependence of the Bessel function, $J_{0}(q b)=$ $1-\frac{1}{4} q^{2} b^{2}+\cdots$ introduces a term proportional to $b^{2}$ in first order, and shifts the peak to a location determined by the logarithmic slope parameter $B$, with

$$
\begin{aligned}
f_{N, c}\left(s, q^{2}\right) \approx & \left(\left.e^{2 i \delta_{c}(b, s)+2 i \delta_{c}^{F F}(b, s)}\right|_{b=\sqrt{\sigma_{\mathrm{tot}}(s) / 4 \pi}}\right. \\
& \left.+\left.\frac{1}{2} q^{2} B e^{2 i \delta_{c}(b, s)+2 i \delta_{c}^{F F}(b, s)}\right|_{b=\sqrt{B(s)}}\right) \\
& \times f_{N}\left(s, q^{2}\right)+O\left(q^{4}\right)
\end{aligned}
$$

in the Bethe approximation.

Rather than using this approximation, our approach has instead been to use our existing eikonal fit to $p p$ scattering [14], evaluate the integral in Eq. (27) directly, and then compare the results to a direct evaluation of the nuclear amplitude itself, Eq. (7). Any fit to the $p p$ scattering amplitude consistent with unitarity can be put in eikonal form, and a similar analysis made. However, we stress that successful fits cannot deviate substantially from our fit, which describes the scattering reasonably well from $5 \mathrm{GeV}$ to the $\mathrm{TeV}$ range. In particular, we calculate the modified amplitude in Eq. (27) numerically using the eikonal model in [22] and relate the result in phase-amplitude form to the pure nuclear amplitude.

Our results for the ratio $\left|f_{N, c}\left(s, q^{2}\right) / f_{N}\left(s, q^{2}\right)\right|$ of the ratio of magnitudes of the nuclear amplitude with Coulomb and form-factor corrections to the pure nuclear amplitude is shown in Fig. 1. As expected, and seen in the upper half of 

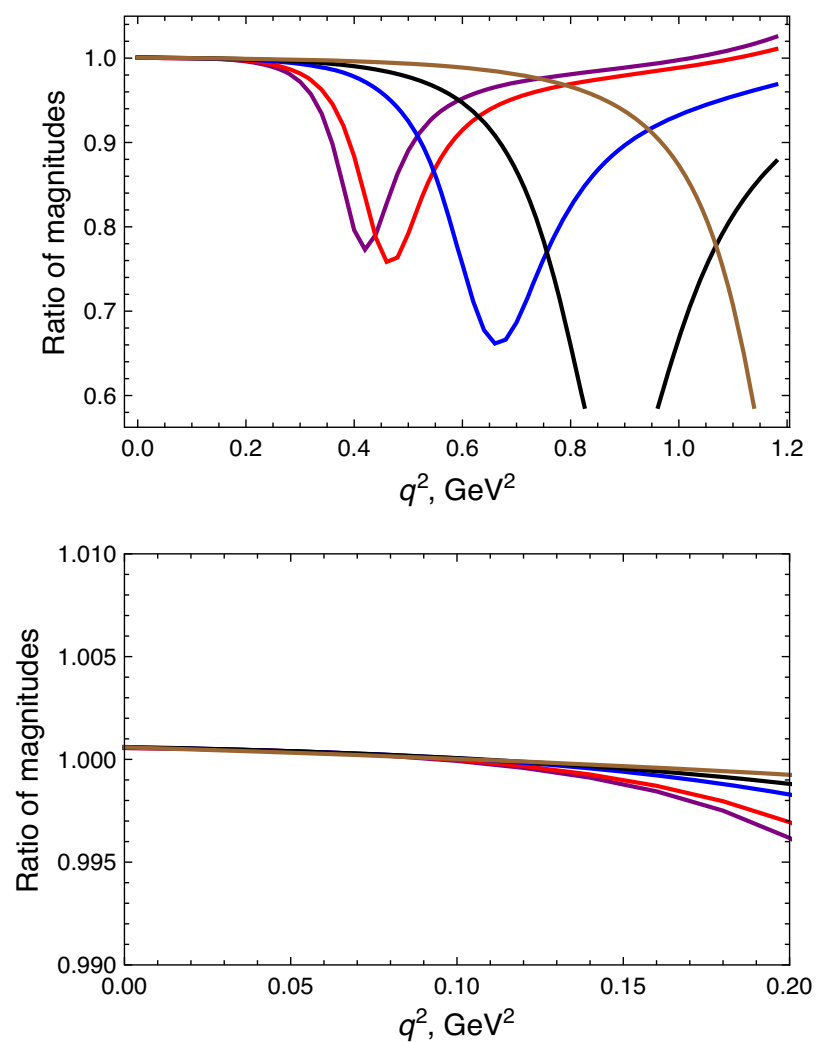

FIG. 1. The ratios of magnitudes $\left|f_{N c}\left(s, q^{2}\right)\right| /\left|f_{N}\left(s, q^{2}\right)\right|$ at 100 (brown), 546 (black), 1800 (blue), 8000, (red) and 13000 (purple) $\mathrm{GeV}$, top to bottom on the right-hand side of the lower figure, top to bottom on the left in the upper figure.

the figure, the ratio is very close to unity at small $q^{2}$ where the amplitudes should differ by a pure phase in the Bethe approximation, but differs substantially at large $q^{2}$ where that approximation fails and the diffraction structure of the amplitudes is important. As seen in the lower half of the figure, the corrections are a small fraction of a percent for $q^{2}<0.2 \mathrm{GeV}^{2}$, the range used in typical analyses, and less that $0.1 \%$ near $q^{2}=0$. We will ignore these corrections, and treat the ratio of amplitudes as a pure phase.

We show the difference $\Delta \Phi_{N}\left(s, q^{2}\right)=\Phi_{N, c}-\Phi_{N}$ between the phases of the corrected and pure nuclear amplitudes,

$$
\begin{aligned}
\Delta \Phi_{N}\left(s, q^{2}\right) & =\arg f_{N, c}\left(s, q^{2}\right)-\arg f_{N}\left(s, q^{2}\right) \\
& =\arg \left(f_{N, c}\left(s, q^{2}\right) / f_{N}\left(s, q^{2}\right)\right)
\end{aligned}
$$

in Fig. 2. The energy dependence of this difference is due mostly to the factor $(p b)^{2 i \eta}$ in the Coulomb phase in Eq. (27); the same dependence on $p$ appears in the Coulomb and form-factor term in Eq. (21), and will drop out in the differential cross section.

The dependence of $\Delta \Phi_{N}\left(s, q^{2}\right)$ on $s$ and $q^{2}$ is described very well by an expression quadratic in $q^{2}$ and linear in the momentum $p(W)$,

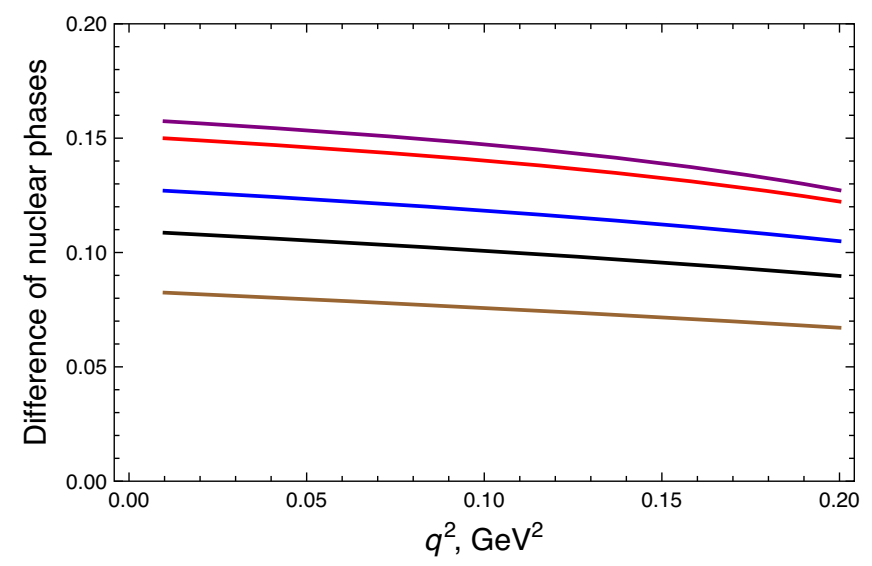

FIG. 2. The differences of nuclear phases $\Delta \Phi_{N}\left(s, q^{2}\right)=$ $\arg \left(f_{N, c}\left(s, q^{2}\right)\right)-\arg \left(f_{N}\left(s, q^{2}\right)\right)$ at (top to bottom) at 13000 (purple), 8000 (red), 1800 (blue), 546 (black), and 100 (brown) GeV.

$$
\begin{aligned}
\Delta \Phi_{N}\left(s, q^{2}\right) \approx & a_{1}+a_{2} q^{2}+a_{3} q^{4} \\
& +\left(b_{1}+b_{2} q^{2}+b_{3} q^{4}\right) \log p .
\end{aligned}
$$

The parameters in the fit are given in Table I.

We compare these results with those obtained in the Bethe approximation in Fig. 3. While differences do not appear to be large, they are significant on the scale of the final phase differences in Fig. 4. In that figure, we show the total phase difference $\Phi_{\text {tot }}\left(s, q^{2}\right)$ between the Coulomb and nuclear parts of the scattering amplitude when it is written so that the Coulomb term is real with the phase $\left(4 p^{2} / q^{2}\right)^{i \eta}$ absorbed,

$$
\begin{gathered}
f\left(s, q^{2}\right)=-\frac{2 \eta}{q^{2}} F^{2}\left(q^{2}\right)+e^{i \Phi_{\mathrm{tot}}\left(s, q^{2}\right)} f_{N}\left(s, q^{2}\right), \\
\Phi_{\mathrm{tot}}\left(s, q^{2}\right)=\Phi_{N, c}\left(s, q^{2}\right)-\Phi_{N}\left(s, q^{2}\right)-\Phi_{c, F F}\left(s, q^{2}\right) .
\end{gathered}
$$

The main energy dependence of $\Phi_{N, c}\left(s, q^{2}\right)$ through the factor $p^{2 i \eta}$ in the integrand for $f_{N, c}\left(s, q^{2}\right)$, Eq. (27), cancels with the corresponding factor in $\Phi_{c, F F}$; the residual energy dependence arises from that in the nuclear part of the integrands.

TABLE I. The parameters in the fit in Eq. (30) to the phase difference.

\begin{tabular}{lc}
\hline \hline Parameter & Value, radians \\
\hline$a_{1}$ & 0.02346 \\
$a_{2}$ & -0.08661 \\
$a_{3}$ & 0.34517 \\
$b_{1}$ & 0.01530 \\
$b_{2}$ & 0.00280 \\
$b_{3}$ & -0.08678 \\
\hline \hline
\end{tabular}




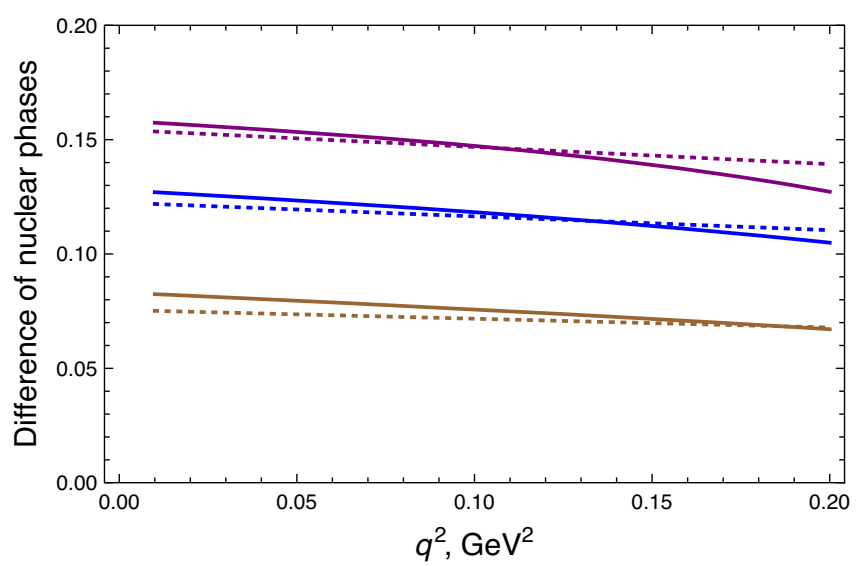

FIG. 3. Phases in the Bethe approximation of Eq. (28) (dashed curves) compared to the exact phases (solid curves) at, top to bottom, 13000 (purple), 1800 (blue), and 100 (brown) GeV.

The form of the amplitude in Eq. (31) is particularly convenient. The Coulomb amplitude is real, so the only source of Coulomb-nuclear interference in the differential cross section is through the real part of the nuclear term. The phase $\Phi_{\text {tot }}$ is quite small as seen in Fig. 4, so the main effect of the phase factor $e^{i \Phi_{\mathrm{tot}}\left(s, q^{2}\right)}=1+$ $i \Phi_{\text {tot }}\left(s, q^{2}\right)+\cdots$ is to mix a small component of $\Im f_{N}\left(s, q^{2}\right)$ into the real part $\Re f_{N}\left(s, q^{2}\right)$. While fractionally small, it is still significant because $\Im f \gg \Re f$ in the interference region. This structure is not immediately evident when the Coulomb and nuclear phases are included separately on those terms. The change in the imaginary part of the amplitude is small, given by the product of $\Re f_{N}$ and $\Phi_{\text {tot }}$.

Separating out these correction terms, we can write the complete amplitude as

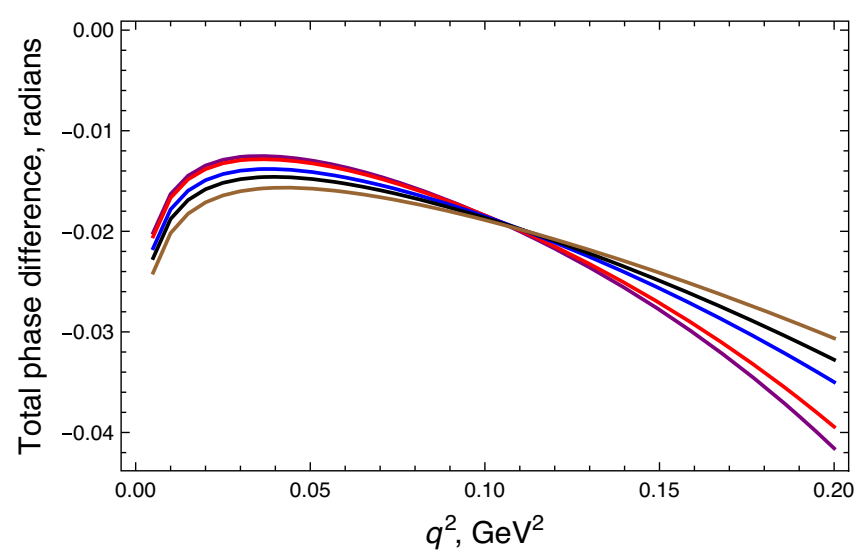

FIG. 4. Total phase differences $\Phi_{\text {tot }}\left(s, q^{2}\right)$, Eq. (32), between the nuclear and Coulomb amplitudes at 100 (brown), 546 (black), 1800 (blue), 8000 (red), and 13000 (purple) GeV, top to bottom on the right.

$$
\begin{aligned}
f\left(s, q^{2}\right)= & -\frac{2 \eta}{q^{2}} F^{2}\left(q^{2}\right)-\Phi_{\mathrm{tot}}\left(s, q^{2}\right) \Im f_{N}\left(s, q^{2}\right) \\
& +i \Phi_{\mathrm{tot}}\left(s, q^{2}\right) \Re f_{N}\left(s, q^{2}\right)+f_{N}\left(s, q^{2}\right) .
\end{aligned}
$$

In this form, the pure nuclear amplitude is displayed separately. However, it is important to recognize that the corrections will affect any attempt to determine $f_{N}$ by fitting data away from the main Coulomb-nuclear interference region, and must be taken into account, as must the tail of the Coulomb term.

The effect of the correction is illustrated in Fig. 5, where we compare the real parts of the nuclear amplitude with and without the phase correction. The final correction is not large. A reasonable first approximation is in fact to ignore the correction and take the full amplitude simply as the sum of the (real) leading-order Coulomb term with form factors and the uncorrected nuclear amplitude. However, in the present form, the corrections are very simple to include, and should be used. The simplicity is striking relative to the treatment of the corrections in $[3,5]$.

We emphasize that the corrections shown in Fig. 4 are very stable and do not change for reasonable changes in the eikonal model. This is to be expected: in the Bethe approximation, the corrections to the nuclear phase are independent of the details of the model, and require only that the eikonal amplitude be strongly peaked in impact parameter space. This is a generic feature of realistic scattering amplitudes at high energies. We note also that the corrections depend mostly on the imaginary part of the nuclear impact parameter distribution, which is dominant at high energies and well determined by fits to the total cross section and the slope parameter $B$. We give an example of an alternative model in the next section; the changes in the corrections are indiscernible in the equivalent of Fig. 4.

In Fig. 6 we show the effects of the Coulomb-nuclear interference on the final cross section, plotting the ratio

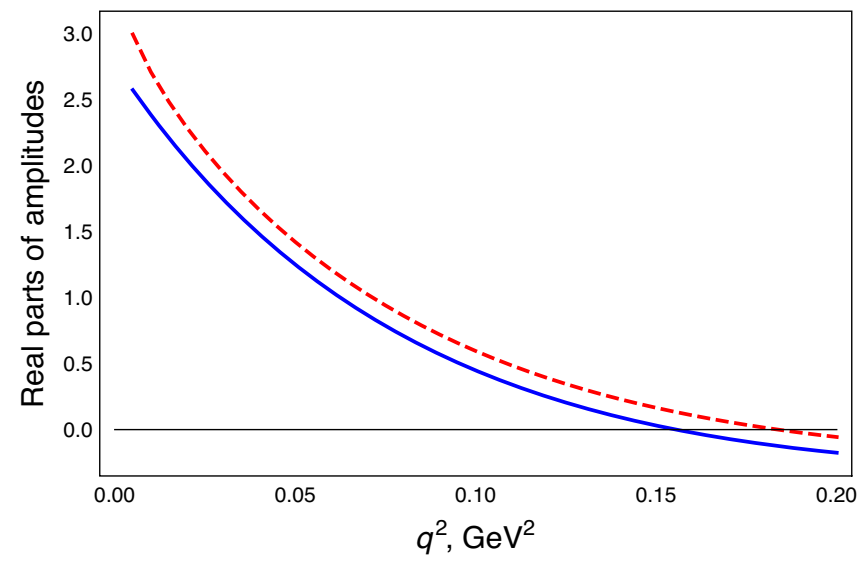

FIG. 5. Comparison of the real part of the actual nuclear amplitude at $13 \mathrm{TeV}$ (solid blue curve) with the effective real part including the Coulomb and electromagnetic form-factor corrections, Eqs. (31) and (32) (dashed red curve). 


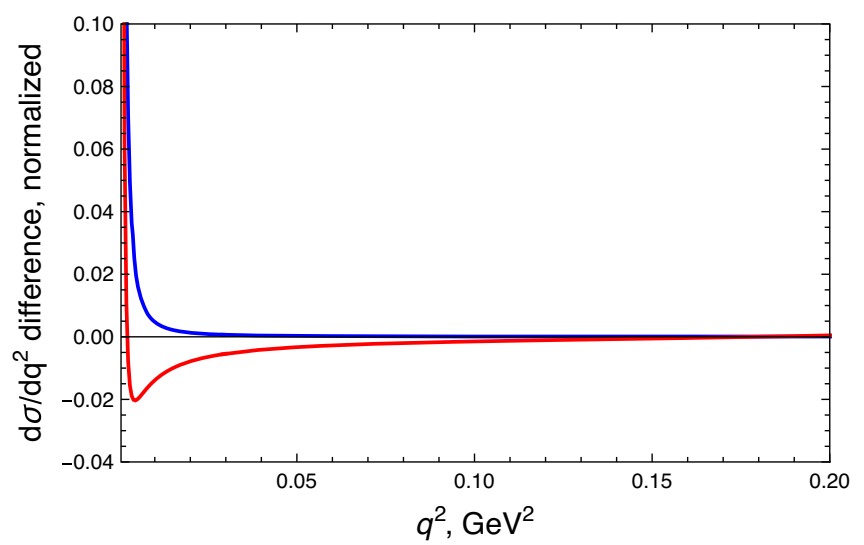

FIG. 6. Plots of the cross section ratio $\left(d \sigma / d q^{2}-d \sigma_{N} / d q^{2}\right) /$ $\left(d \sigma_{N} / d q^{2}\right)$ (lower red curve) and the corresponding ratio with the Coulomb-nuclear interference term dropped (upper blue curve) in $p p$ scattering in the eikonal model at $13 \mathrm{TeV}$.

$\left(d \sigma / d q^{2}-d \sigma_{N} / d q^{2}\right) /\left(d \sigma_{N} / d q^{2}\right)$ as a function of $q^{2}$ at $13 \mathrm{TeV}$ (lower red curve). We also show the ratio of cross sections in the absence of interference, $\left(d \sigma_{c} / d q^{2}+\right.$ $\left.d \sigma_{N} / d q^{2}\right) /\left(d \sigma_{N} / d q^{2}\right)-1=\left(d \sigma_{c} / d q^{2}\right) /\left(d \sigma_{N} / d q^{2}\right)$ (upper blue curve), where $d \sigma_{c} / d q^{2}$ is the Coulomb cross section. The interference effects are small, at most $2 \%$ at the dip at $q^{2}=0.0045 \mathrm{GeV}^{2}$, that is, of order $\eta \approx \alpha$, and are significant only at very small values of $q^{2}$. This is the result of the $1 / q^{2}$ falloff of the Coulomb amplitude further suppressed by the effects of the proton charge form factors. For comparison, the statistical uncertainties in the measured cross sections in [13] vary from $\sim 0.5 \%$ at the dip to $\sim 1 \%$ for $q^{2} \approx 0.2$. The results are similar at lower energies, with the only significant sensitivity in the region of the dip.

\section{AN APPLICATION AND SIMPLE MODEL FOR THE AMPLITUDES}

As an application of these results, we consider a model which has been used frequently in the analysis of experimental data, e.g., the TOTEM data at 8 and $13 \mathrm{TeV}$; see $[13,15]$ and earlier references therein. In this model, the phase of the nuclear amplitude is taken as a constant independent of $q^{2}$. It is determined simply by the ratio $\rho$ of the real to the imaginary parts of the nuclear amplitude in the forward direction, corresponding to a phase $\Phi_{N}\left(s, q^{2}\right) \equiv$ $\frac{\pi}{2}-\arctan \rho(s)$ and $f_{N}\left(s, q^{2}\right)=e^{i \Phi_{N}}\left|f_{N}\left(s, q^{2}\right)\right|$. This is clearly unrealistic in general, but may be adequate in a small region near $q^{2}=0$.

We will consider two versions of this model. In the first, we write the complex eikonal amplitude in Eq. (2) approximately in terms of its dominant imaginary part multiplied by a constant phase and properly normalized,

$$
\begin{aligned}
i(1 & \left.-e^{2 i\left(\delta_{c}^{\text {tot }}+\delta_{N}\right)}\right) \\
& \rightarrow i e^{-i \arctan \rho} \Im i\left(1-e^{2 i\left(\delta_{c}^{\text {tot }}+\delta_{N}\right)}\right) / \cos (\arctan \rho) .
\end{aligned}
$$

This form allows us to calculate the Coulomb and formfactor corrections to the nuclear phase. As discussed above, the results are essentially identical to those obtained using the full amplitude shown in Fig. 4 even though the real parts of the amplitudes and the cross sections differ. This is as expected given the Bethe argument.

Since the corrections are effectively model independent, we can proceed to a simpler construction used in various experimental analyses and write the constant-phase amplitude approximately in terms of the standard small- $q^{2}$ expansion of the nuclear scattering cross section,

$$
\frac{d \sigma}{d q^{2}}\left(s, q^{2}\right) \approx A e^{-B q^{2}+C q^{4}-D q^{6}+\cdots}, \quad 0 \leq q^{2} \ll 1,
$$

where $B$ is the usual slope parameter and $C, D, \ldots$ introduce curvature in $d \sigma / d q^{2}$. Taking the square root and introducing a phase, we have

$$
\sqrt{\pi} f_{N}\left(s, q^{2}\right) \approx \sqrt{A} e^{i \Phi_{N}} e^{-\frac{1}{2}\left(B q^{2}-C q^{4}+D q^{6}-\cdots\right)} .
$$

We will initially take $\Phi_{N}$ as constant, with $\Phi_{N}=$ $\frac{\pi}{2}-\arctan \rho$. This is the form assumed, for example, in the TOTEM analyses of Coulomb-nuclear interference $[13,15]$, with $\rho$ used as a parameter in fitting the data in the interference region. Note that this form, with $\Phi_{N}$ constant, does not allow for zeros and the associated changes in sign of the real and imaginary parts of the amplitude as at the diffraction zeros in $f_{N}$, so is restricted to small $q^{2}$.

The expansion in Eq. (35) and its range of validity were investigated in detail in [23], where exact expressions were given for the parameters $B, C$, and $D$ in the eikonal approach. As noted there, the predicted values of those parameters were consistent with the results obtained by the TOTEM collaboration in their fits to their $\mathrm{TeV}$ data [24]. The next term in the series becomes important near the upper end of the range of $q^{2}$ used in the TOTEM fits, with errors in the fitted cross section comparable to, or larger than, the uncertainties in the experimental results.

In general, fits based on Eq. (35) should use $q^{2} \lesssim$ $0.1-0.15 \mathrm{GeV}^{2}$ at the higher energies; TOTEM used values of $q^{2}$ up to $0.2 \mathrm{GeV}^{2}$. This use of a too-wide range of $q^{2}$ is also common in analyses at lower energies; corrections to the quoted results were considered in detail in [22].

It is straightforward to estimate the value of the next coefficient in the series using the calculated value of the local slope parameter $B\left(q^{2}\right)$ [23] at a small value of $q^{2}$ such as $q_{0}^{2}=0.01 \mathrm{GeV}^{2}$. Since $B\left(q_{0}^{2}\right)=$ $-d \log \left(d \sigma / d q^{2}\right) /\left.d q^{2}\right|_{q_{0}^{2}} \approx$

$B-2 C q_{0}^{2}+3 D q_{0}^{4}-4 E q_{0}^{6}+O\left(q_{0}^{8}\right)$, we can express $E$ in terms of $B\left(q_{0}^{2}\right)$ and the known values of $B, C$, and $D$. We will not use this refinement here, though it extends the range of validity of the expansion to approximately that 
used in $[13,15,24]$, but will follow the procedures used there and simply fit the exact eikonal amplitudes at 8 and $13 \mathrm{TeV}$ using the expression in Eq. (36). The fitted values of $A$ and $B$ do not differ significantly from the exact values. $C$ changes by a few percent, and $D$ changes significantly. The results are consistent with those found by the TOTEM collaboration.

We compare the results for $\Re f_{N}\left(s, q^{2}\right)$ obtained using the fits and Eq. (36) with the exact eikonal results at 8 and $13 \mathrm{TeV}$ in Fig. 7. The real parts in the constant phase approximation (top blue curves) are systematically larger than the exact results (bottom red curves), suggesting that this approach will lead to reduced values of $\rho$ when used to fit data. A better approximation is needed.

As is evident from Fig. 5, the real part of the nuclear amplitude drops rapidly with increasing $q^{2}$, and actually changes sign in the region used in the TOTEM analyses. We therefore propose a simple approximation for the phase which takes this behavior into account. Since $\Phi_{N}\left(s, q^{2}\right)=\frac{\pi}{2}-\arctan \rho\left(s, q^{2}\right)$, we concentrate on $\rho\left(s, q^{2}\right)=\Re f_{N}\left(s, q^{2}\right) / \Im f_{N}\left(s, q^{2}\right)$. At high energies, $\Re f_{N}$ has a zero at small $q^{2}, \Re f_{N}\left(s, q_{R}^{2}\right)=0$. Similarly, $\Im f_{N}\left(s, q_{I}^{2}\right)=0$ at the first diffraction dip in $d \sigma / d q^{2}$ at
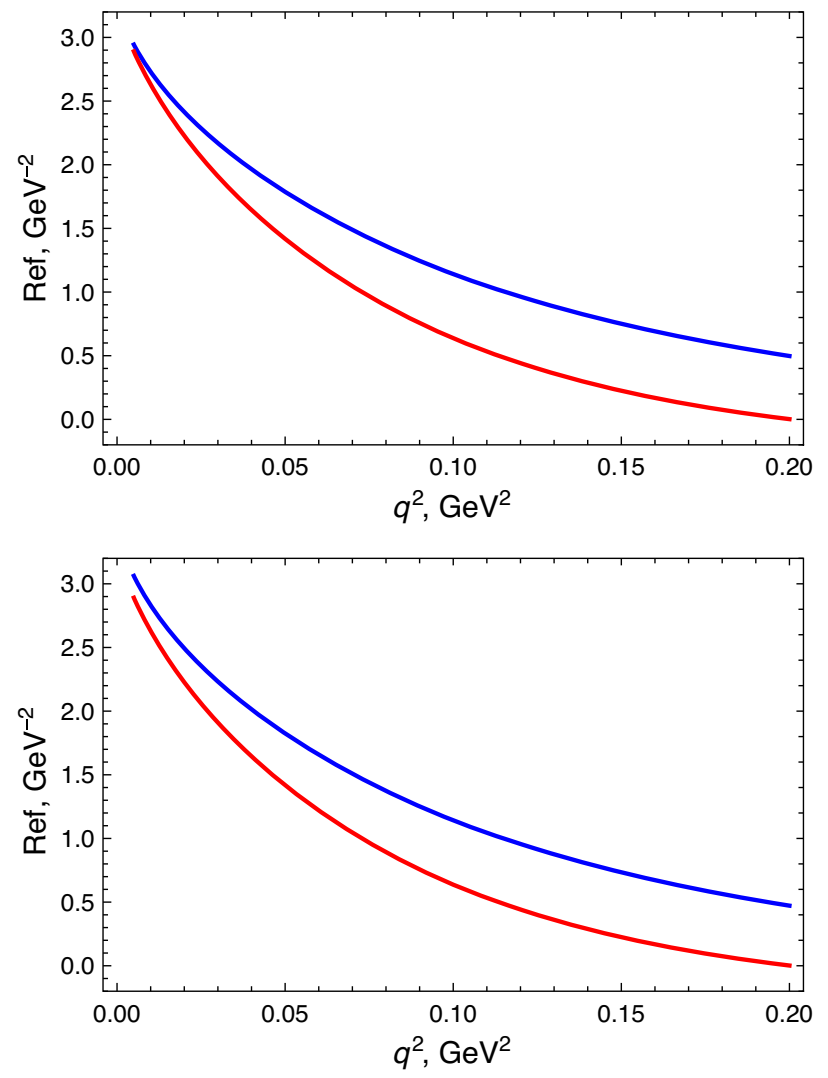

FIG. 7. Comparison of the real part of the nuclear amplitude with Coulomb and form-factor corrections included (bottom red curves) with the corresponding real part of the amplitude in the constant phase approximation (top blue curves) at $\mathrm{W}=8 \mathrm{TeV}$ (top figure) and $13 \mathrm{TeV}$ (bottom figure).
$q_{I}^{2}>q_{R}^{2}$ [25]. Taking these zeros into account, we write

$$
\rho\left(s, q^{2}\right) \approx \rho(s) \frac{1-q^{2} / q_{R}^{2}}{1-q^{2} / q_{I}^{2}} .
$$

This form reduces to $\rho=\rho(s)$ at $q^{2}=0$ and has the proper zeros built in, with $\Phi_{N}\left(s, q^{2}\right)=\pi / 2$ at $q_{R}^{2}$ and $f_{N}\left(s, q_{R}^{2}\right)$ purely imaginary, and $\Phi_{N}=0$ at $q_{I}^{2}$ and $f_{N}$ real. The value of $\rho$ can again be used as a fitting parameter.
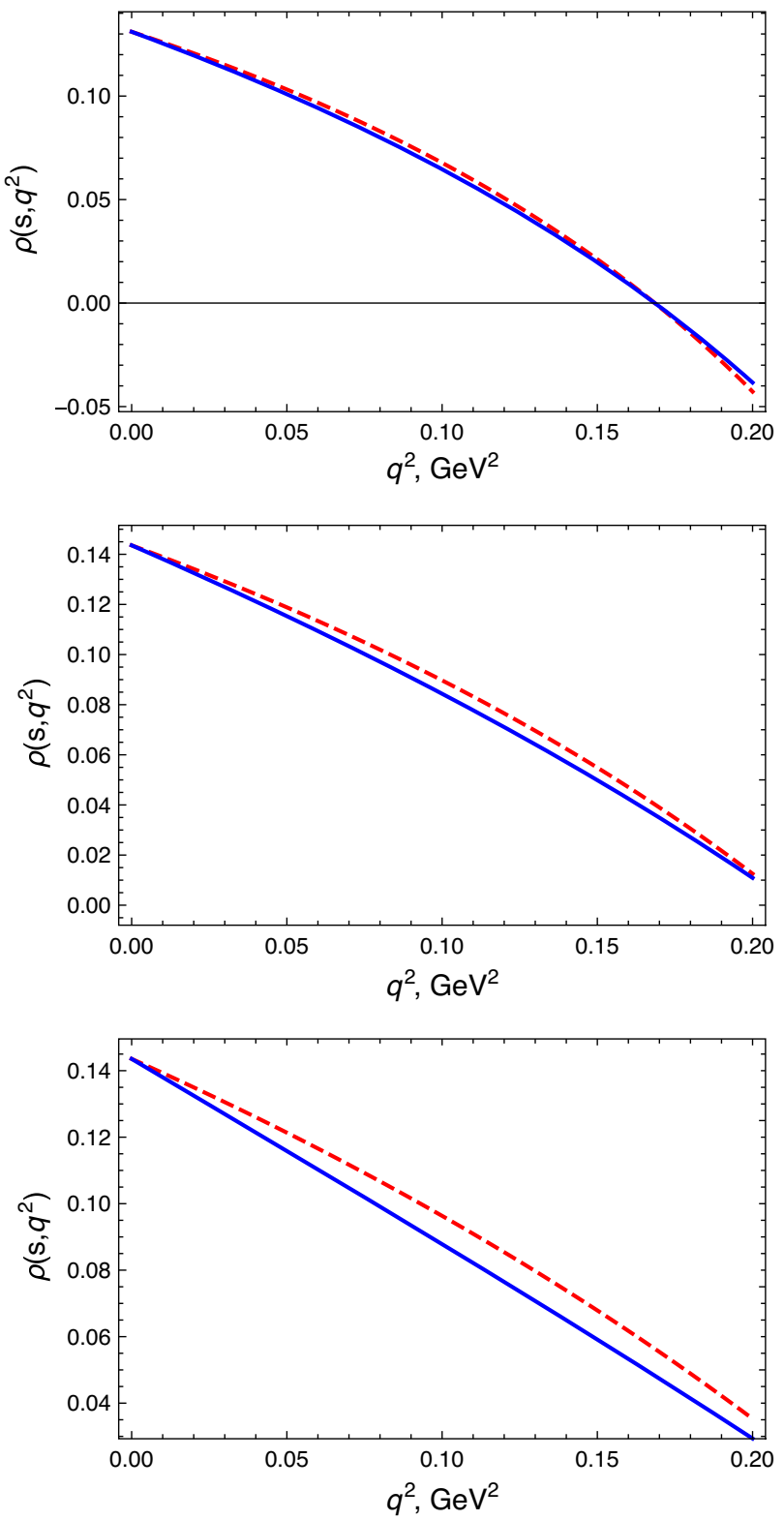

FIG. 8. Comparison of the values of the ratio $\rho\left(q^{2}\right)=$ $\Re f_{N}\left(q^{2}\right) / \mathfrak{I} f_{N}\left(q^{2}\right)$ calculated in the eikonal model at 8000 , 1800 , and $546 \mathrm{GeV}$, top to bottom, with the approximate values given in terms of the zeros of the real and imaginary parts of the amplitude by Eq. (37). The actual values are given by the solid blue curves, approximate values, by the dashed red curves. 

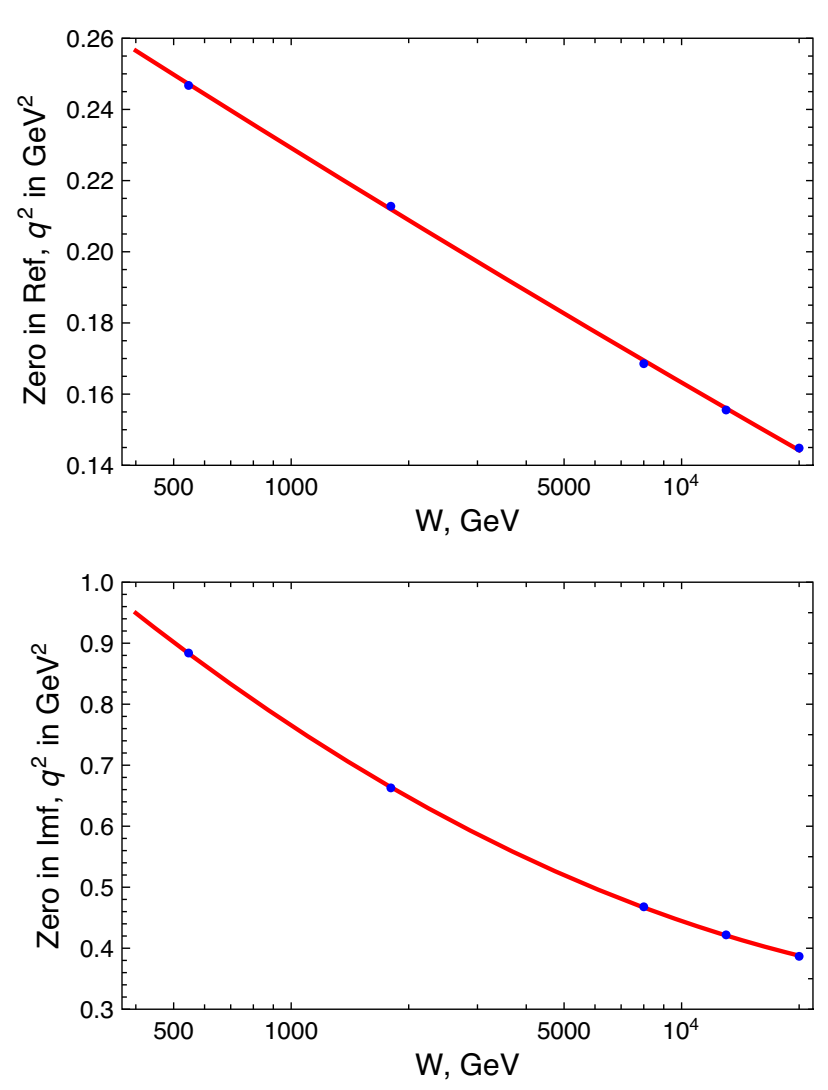

FIG. 9. Location in $q^{2}$ of the zeros in the real and imaginary parts of the nuclear amplitude in the eikonal model as functions of $W$. The points give the actual values of $q^{2}$ at the zeros in the eikonal model; the lines correspond to the fits in the text.

We compare the actual and approximate values of $\rho\left(s, q^{2}\right)$ in the eikonal model [14] at 8000, 1800, and $546 \mathrm{GeV}$ in Fig. 8. The approximate results-and the corresponding results for $\Re f_{N}\left(s, q^{2}\right)$ - are quite accurate at the higher energies, and still good in the $500 \mathrm{GeV}$ region. They are much better than the results obtained with the so-called "standard phase" used in some analyses which takes only the diffraction zero in the imaginary part of the amplitude into account (Sec. 6.1.3 of [13]), and then only approximately.

We note that the errors in Fig. 8 can be essentially eliminated by multiplying the expression in Eq. (37) by a factor $\left(1-a q^{2}\right)$ with an appropriate value of the coefficient $a$. This is useful in obtaining accurate fits to $\rho\left(s, q^{2}\right)$. However, the approximate expression in Eq. (37) requires knowledge only of the location of the zeros in $\Re f_{N}$ and $\Im f_{N}$. In particular, $q_{I}^{2}$ can be estimated from the diffraction structure of the cross section, while, roughly, $q_{R}^{2} \approx q_{I}^{2} / 3$ at high energies in the eikonal model.

The actual location of the zeros in the real and imaginary parts of $f_{N}\left(s, q^{2}\right)$ in the eikonal model [14] are plotted in Fig. 9. The curves in the figure correspond to a fit with
TABLE II. The parameters in the fit to the locations in $q^{2}$ of the zeros in the real and imaginary parts of the eikonal scattering amplitude in $W$ given in Eq. (38).

\begin{tabular}{lc}
\hline \hline Parameter & Value, $\mathrm{GeV}^{2}$ \\
\hline$a_{R}$ & 0.4514 \\
$b_{R}$ & -0.03484 \\
$c_{R}$ & 0.000386 \\
$a_{I}$ & 2.9464 \\
$b_{I}$ & -0.4481 \\
$c_{I}$ & 0.01916 \\
\hline \hline
\end{tabular}

$$
\begin{aligned}
& q_{R}^{2}(W)=a_{R}+b_{R} \log W+c_{R} \log ^{2} W \\
& q_{I}^{2}(W)=a_{I}+b_{I} \log W+c_{I} \log ^{2} W .
\end{aligned}
$$

The parameters in the fit are given in Table II. We note that the fit becomes inaccurate at energies below a few hundred $\mathrm{GeV}$, where the zeros are displaced by small contributions from exchange terms dependent on inverse powers of $W$; see e.g., [14].

These results can be used in conjunction with the expansion in Eq. (36) to construct nuclear amplitudes with a realistic $q^{2}$ dependence and phase at small $q^{2}$, again retaining $\rho=\rho(s)$ as a parameter to be used in fitting data. With the phase $\Phi_{N}=\frac{\pi}{2}-\arctan \rho\left(s, q^{2}\right)$ calculated using the expression for $\rho$ in Eq. (37), the diffraction zeros in the real and imaginary parts of the amplitude are built in, and the magnitude of the amplitude is simply $\sqrt{d \sigma / d q^{2}}$.

\section{CONCLUSIONS}

We have presented a very simple way of calculating Coulomb and form-factor corrections to the $p p$ scattering amplitude in the context of an eikonal model. As we have emphasized, our approach is much simpler than that of Cahn [3] and Kundrát and Lokajiček [5] which seem to have become standard in the analysis of Coulomb-nuclear interference effects at high energies. It is essentially model independent, with the corrections holding for any reasonable eikonal model which fits the total $p p$ scattering cross section and the forward slope parameter $B$ and gives a reasonable description of $d \sigma / d q^{2}$.

We have given parametrizations of corrections that hold at least from $100 \mathrm{GeV}$ to $20 \mathrm{TeV}$, and illustrated the magnitude of the effects in the effective real part of the nuclear amplitude which interferes with the Coulomb amplitude in an appropriate phase convention. We have used the results to investigate the constant-phase approximation used in recent analyses of very-high-energy scattering, and introduced a very simple model for the correct $q^{2}$ dependence of the phase based on the location of the zeros in the real and imaginary parts of the nuclear component of the amplitude. 


\section{ACKNOWLEDGMENTS}

L. D. would like to thank the Aspen Center for Physics for its hospitality and for its partial support of this work under NSF Grant No. 1066293. P. H. would like to thank Towson University Fisher College of Science and Mathematics for support.

\section{APPENDIX: THE EFFECTIVE SPIN-AVERAGED EIKONAL AMPLITUDE}

It is generally assumed that spin effects will be very small at very high energies, with the scattering dominated by absorptive effects that are insensitive to spin. However, a complete description of the $p p$ scattering matrix involves a number of spin-dependent amplitudes. These are conveniently labeled by the helicities of the incident and final protons following Jacob and Wick [26]. For a process $a+b \rightarrow c+d$ where the initial and final particles have helicities $\lambda_{a}, \lambda_{b}, \lambda_{c}, \lambda_{d}$, the scattering amplitude $f_{\lambda_{c}, \lambda_{d} ; \lambda_{a}, \lambda_{b}}$ assumes the form

$$
\begin{aligned}
f_{\lambda_{c}, \lambda_{d} ; \lambda_{a}, \lambda_{b}}\left(s, q^{2}\right)= & \frac{i}{2 p} \sum_{j}(2 j+1)\left(\delta_{\lambda_{a} \lambda_{c}} \delta_{\lambda_{b} \lambda_{d}}-S_{\lambda_{c}, \lambda_{d} ; \lambda_{a}, \lambda_{b}}\right) \\
& \times d_{\lambda_{a}-\lambda_{b} ; \lambda_{c}-\lambda_{d}}^{j}(\cos \theta),
\end{aligned}
$$

where $j$ is the total angular momentum and the functions $d_{\lambda_{a}-\lambda_{b} ; \lambda_{c}-\lambda_{d}}^{j}(\cos \theta)$ are the standard rotation coefficients in the convention of Rose [27]. The differential cross sections for specific helicity states are

$$
d \sigma_{\lambda_{c}, \lambda_{d} ; \lambda_{a}, \lambda_{b}} / d q^{2}=\frac{\pi}{p^{2}}\left|f_{\lambda_{c}, \lambda_{d} ; \lambda_{a}, \lambda_{b}}\left(s, q^{2}\right)\right|^{2},
$$

while the spin-averaged differential cross section is

$$
\frac{d \sigma}{d q^{2}}=\frac{\pi}{p^{2}} \frac{1}{\left(2 s_{a}+1\right)\left(2 s_{b}+1\right)} \sum_{\lambda_{c}, \lambda_{d}, \lambda_{a}, \lambda_{b}}\left|f_{\lambda_{c}, \lambda_{d} ; \lambda_{a}, \lambda_{b}}\left(s, q^{2}\right)\right|^{2} .
$$

The number of independent $S$-matrix elements for total angular momentum $j$ is restricted by time reversal $\left(S_{\lambda_{a}, \lambda_{b}: \lambda_{c}, \lambda_{d}}^{j}=S_{\lambda_{c}, \lambda_{d} ; \lambda_{a}, \lambda_{b}}^{j}\right)$, parity $\left(S_{-\lambda_{c},-\lambda_{b} ;-\lambda_{a},-\lambda_{b}}^{j}=S_{\lambda_{c}, \lambda_{d} ; \lambda_{a}, \lambda_{b}}^{j}\right)$, and, for $p p$ scattering, the identity of the particles, $\left(S_{\lambda_{a}, \lambda_{b} ; \lambda_{c}, \lambda_{d}}^{j}=S_{\lambda_{c}, \lambda_{d} ; \lambda_{a}, \lambda_{b}}^{j}\right)$, leaving five independent elements. These are conventionally taken, following Sec. IV of [28], as

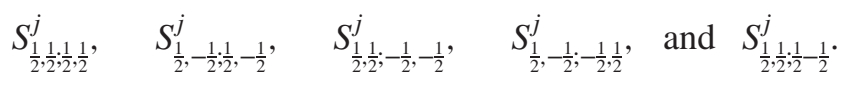

The first two, and the equal corresponding diagonal amplitudes $S_{-\frac{1}{2},-\frac{1}{2} ;-\frac{1}{2},-\frac{1}{2}}^{j}, S_{-\frac{1}{2}, \frac{1}{2} ;-\frac{1}{2}, \frac{1}{2}}^{j}$ involve no helicity flips and are expected to be dominated at high energies by diffractive scattering, with no significant dependence on the helicities involved. Under this condition, those $S$-matrix elements and the corresponding scattering amplitudes are all approximately equal.

The independent diagonal scattering amplitudes are

$$
\begin{aligned}
f_{\frac{1}{2} \cdot \frac{1}{2} \cdot \frac{1}{2} \cdot \frac{1}{2}}\left(s, q^{2}\right) & =\frac{i}{2 p} \sum_{j}(2 j+1)\left(1-S_{\frac{1}{2}, \frac{1}{2}, \frac{1}{2}, \frac{1}{2}}^{j}\right) d_{0,0}^{j}(\cos \theta) \\
& \approx i \int_{0}^{\infty} d b b\left(1-S_{\frac{1}{2}, \frac{1}{2} \cdot \frac{1}{2}, \frac{1}{2}}(s, b)\right) J_{0}(q b), \\
f_{\frac{1}{2},-\frac{1}{2} \cdot \frac{1}{2},-\frac{1}{2}}\left(s, q^{2}\right) & =\frac{i}{2 p} \sum_{j}(2 j+1)\left(1-S_{\frac{1}{2},-\frac{1}{2} \cdot \frac{1}{2},-\frac{1}{2}}^{j}\right) d_{1,1}^{j}(\cos \theta) \\
& \approx i \int_{0}^{\infty} d b b\left(1-S_{\frac{1}{2},-\frac{1}{2} \cdot \frac{1}{2},-\frac{1}{2}, \frac{\mathrm{A}}{2}}(s, b)\right) J_{0}(q b) .
\end{aligned}
$$

In these expressions, we have converted the sums over $j$ to integrals over the impact parameter $b=\sqrt{j(j+1)} / p$, and used asymptotic relations between the rotation coefficients and Bessel functions derivable from known results on the relation between Jacobi polynomials and Bessel functions for $j$ large (Sec. 8.1 of [29]). These relations are discussed in detail in Secs. IIIA and IIID of [30], where the rotation coefficients are expressed for large $j$ in terms of series of Bessel functions.

In the limit of no significant helicity dependence, the $S$-matrix elements in Eqs. (A6) and (A8) have a common limit $S(b, s)$,

$$
S_{\frac{1}{2}, \frac{1}{2} \cdot \frac{1}{2}, \frac{1}{2}}(s, b) \approx S_{\frac{1}{2},-\frac{1}{2}, \frac{1}{2},-\frac{1}{2}}(s, b) \approx S(s, b) .
$$

It follows that the diagonal scattering amplitudes can all be expressed in terms of a single helicity-independent amplitude $f\left(s, q^{2}\right)$, with

$$
\begin{aligned}
f_{\frac{1}{2}, \frac{1}{2} \cdot \frac{1}{2}, \frac{1}{2}}\left(s, q^{2}\right) & =f_{-\frac{1}{2},-\frac{1}{2} ;-\frac{1}{2},-\frac{1}{2}}\left(s, q^{2}\right) \approx f_{\frac{1}{2},-\frac{1}{2} ; \frac{1}{2},-\frac{1}{2}}\left(s, q^{2}\right) \\
& =f_{-\frac{1}{2}, \frac{1}{2} \cdot \frac{1}{2},-\frac{1}{2}, \frac{1}{2}}\left(s, q^{2}\right) \approx f\left(s, q^{2}\right),
\end{aligned}
$$

where

$$
f\left(s, q^{2}\right)=i \int_{0}^{\infty} d b b(1-S(s, b)) J_{0}(q b) .
$$

Neglecting the presumably very small helicity-flip amplitudes, the spin-averaged differential cross section in Eq. (A3) becomes simply 


$$
\frac{d \sigma}{d q^{2}} \approx \pi\left|f\left(s, q^{2}\right)\right|^{2}
$$

This was the form used in the text, and used without discussion in most treatments of Coulomb-nuclear interference at high energies. In the limit described, it is not necessary to distinguish the two independent diagonal amplitudes, and the off-diagonal elements of the scattering matrix become relevant only when polarization and spin-correlation phenomena, presumably small, are studied, as in [6].

In the absence of Coulomb effects, the total nuclear cross sections for specific initial helicity states are related through standard unitarity arguments and the optical theorem to the imaginary parts of the corresponding diagonal amplitudes $f_{\lambda_{a}, \lambda_{b} ; \lambda_{a}, \lambda_{b}}\left(s, q^{2}\right)$ at $q^{2}=0$,

$$
\sigma_{t o t ; \lambda_{a}, \lambda_{b}}(s)=4 \pi \Im f_{\lambda_{a}, \lambda_{b} ; \lambda_{a}, \lambda_{b}}(s, 0) .
$$

Since, in the limit discussed above, these amplitudes have the common value $f\left(s, q^{2}\right)$, the total cross section for the nuclear scattering averaged over the initial helicities is just

$$
\sigma_{\mathrm{tot}}=4 \pi \Im f_{N}(s, 0),
$$

where we have included the label $N$. This is usual connection.

As emphasized by Buttimore, Gotsman, and Leader in [6], double nuclear helicity-flip amplitudes can interfere with the corresponding magnetic interactions between the protons, and, if large enough, can potentially disrupt the extraction of the $\rho$ parameter from interference effects in the small- $q^{2}$ scattering region when $\rho$ is small. It is therefore useful to estimate the size of these helicity-dependent amplitudes.

In the high-energy regime with which we are concerned, the leading contributions to those amplitudes are expected to arise mainly from the Regge exchange amplitudes associated with the $\rho$ and $\omega$ trajectories. The presence of these exchanges in $p p$ scattering is well established, and the magnitude of the exchange amplitudes can be determined from the total cross sections. It is known, in particular, that the amplitudes decrease in magnitude with increasing energy as $s^{\alpha(0)-1}$ with $\alpha(0) \approx 1 / 2$ the Regge intercept at $q^{2}=0$.

The complete helicity-dependent Regge amplitudes for these exchanges can be constructed using the methods of King, Durand, and Wali [31]. The helicity structure is essentially determined by the leading physical resonance associated with the trajectory, that is, the vector $\rho$ and $\omega$ mesons in the present case. These amplitudes therefore have the same structure as the electromagnetic interactions [18], and can interfere with them.

From the results on the exchange amplitudes in [14], we find that the real parts of those amplitudes at $q^{2}=0$ are about $70 \%$ of the real part of the spin-independent amplitude at $W=50 \mathrm{GeV}$; this ratio decreases to $8 \%$ at $100 \mathrm{GeV}$, and $0.3 \%$ at $1 \mathrm{TeV}$. We expect the magnetic parts of the exchange amplitudes to have similar magnitudes. The expected interference effects between the exchange amplitudes and the electromagnetic magnetic-moment amplitude should be reduced by similar factors relative to the Coulomb-nuclear interference term in the charge sector. The contributions to the cross sections are further reduced at small $q^{2}$ by the factor $q^{2} / 4 m^{2}$ familiar for pure magnetic moment scattering. We conclude that these effects are negligible at high energies and that the potential problem noted in [6] does not actually exist at these energies. The magnetic scattering can therefore be neglected, as in the main text.

The estimated contribution of the helicity-dependent exchange amplitudes themselves to the cross section through either single- or double helicity-flip terms is also small, on the order of $4 \%$ at $10 \mathrm{GeV}$, decreases rapidly with increasing energy, and can also be neglected.
[1] H. A. Bethe, Ann. Phys. (N.Y.) 3, 190 (1958).

[2] G. B. West and D. R. Yennie, Phys. Rev. 172, 1413 (1968).

[3] R. Cahn, Z. Phys. C 15, 253 (1982).

[4] M. M. Islam, Phys. Rev. 162, 1426 (1967).

[5] V. Kundrát and M. Lokajiček, Z. Phys. C 63, 619 (1994).

[6] N. H. Buttimore, E. Gotsman, and E. Leader, Phys. Rev. D 18, 694 (1978).

[7] B. Z. Kopeliovich and A. V. Tarasov, Phys. Lett. B 497, 44 (2001).

[8] O. V. Selyugin, Mod. Phys. Lett. A 11, 2317 (1996).

[9] O. V. Selyugin, Phys. Rev. D 60, 074028 (1999).

[10] V. A. Petrov, Eur. Phys. J. C 78, 221 (2018).
[11] V. A. Petrov, Eur. Phys. J. C 78, 414 (2018).

[12] J. Kašpar, arXiv:2001.10227v1.

[13] G. Antchev et al. (TOTEM Collaboration), Eur. Phys. J. C 76, 661 (2016).

[14] M. M. Block, L. Durand, P. Ha, and F. Halzen, Phys. Rev. D 92, 014030 (2015).

[15] G. Antchev et al. (TOTEM Collaboration), Eur. Phys. J. C 79, 785 (2019).

[16] R. J. Glauber, in Lectures in theoretical Physics : lectures delivered at the Summer Institute for Theoretical Physics, University of Colorado, Boulder, Vol. 1, edited by W. E. Brittin and L.G. Dunham (Interscience Publishers, New York, 1959), pp. 315-414. 
[17] In the Glauber construction [16], the Coulomb interaction $V(\mathbf{r})$ appears as a convolution of the finite charge distributions of the protons with the Coulomb denominator, with a Glauber phase

$$
\begin{aligned}
& \int_{-\infty}^{\infty} d z V_{c}(\mathbf{r}) \\
& \quad=\alpha \int_{-\infty}^{\infty} d z \int d^{3} r_{1} \int d^{3} r_{2} \rho\left(r_{1}\right) \frac{1}{\left|\mathbf{r}-\mathbf{r}_{1}+\mathbf{r}_{2}\right|} \rho\left(r_{2}\right) .
\end{aligned}
$$

After introducing transverse and longitudinal coordinate $\mathbf{b}_{i}, z_{i}$, integrating over the $z$ 's, and applying the Fourier-Bessel transform in the Glauber construction, the leading term in the Glauber amplitude reduces to the expected form in momentum space, $\left(\alpha / q^{2}\right) F^{2}\left(q^{2}\right)$, where the form factors $F\left(q^{2}\right)$ are Fourier transforms of the nonrelativistic charge distributions $\rho$. [In the relativistic theory, $F_{Q}\left(q^{2}\right)$ can be identified as a three-dimensional Fourier transform of the time-averaged charge distribution in the Breit or brick-wall coordinate frame [18]] To isolate the effects of the form factors in the eikonal function, we will rewrite $V_{c}$ as $\alpha / r-\left[\alpha / r-V_{c}(\mathbf{r})\right]$, isolating the long-range part of the Coulomb interaction in the first term. The second term, which vanishes for $r \rightarrow \infty$, gives the phase $2 \delta_{c}^{F F}$ in Eq. (3), the first term, a pure Coulomb phase. The nuclear phase shift adds on separately as in Eq. (2). The connection between the eikonal approach and the Born series for the scattering amplitude is discussed in [16].

[18] L. Durand III, P. C. DeCelles, and R. B. Marr, Phys. Rev. 126, 1882 (1962).

[19] R. G. Newton, Scattering Theory of Waves and Particles (McGraw-Hill Book Company, New York, 1966).
[20] NIST Digital Library of Mathematical Functions, edited by F. W. J. Olver, A. B. Olde Daalhuis, D. W. Lozier, B. I. Schneider, R. F. Boisvert, C. W. Clark, B. R. Miller, B. V. Saunders, H. S. Cohl, and M. A. McClain, https://dlmf.nist .gov/, Release 1.0.24 of 2019-09-15 (2019).

[21] G. N. Watson, Theory of Bessel Functions (Cambridge University Press, Cambridge, England, 1958).

[22] L. Durand and P. Ha, Phys. Rev. D 99, 014009 (2019).

[23] M. M. Block, L. Durand, P. Ha, and F. Halzen, Phys. Rev. D 93, 114009 (2016).

[24] G. Antchev et al. (TOTEM Collaboration), Nucl. Phys. B899, 527 (2015).

[25] The existence of the diffraction zeros in the $p p$ cross section was first predicted over fifty years ago-in the eikonal context-in [32,33], with diffraction effects on the spin polarization also noted in [32].

[26] M. Jacob and G. C. Wick, Ann. Phys. (N.Y.) 7, 404 (1959).

[27] M.E. Rose, Elementary Theory of Angular Momentum (Wiley, New York, 1957).

[28] M. L. Goldberger, M. T. Grisaru, S. W. MacDowell, and D. Y. Wong, Phys. Rev. 120, 2250 (1960).

[29] G. Szegó, Orthogonal Polynomials (American Mathematical Society, New York, 1939), Chap. IV.

[30] L. Durand, J. Math. Phys. (N.Y.) 60, 013501 (2019).

[31] M. J. King, L. Durand, and K. C. Wali, Phys. Rev. D 13, 1409 (1976).

[32] L. Durand and R. Lipes, Phys. Rev. Lett. 20, 637 (1968).

[33] T. T. Chou and C. N. Yang, Phys. Rev. Lett. 20, 1213 (1968). 